\title{
RIESZ CAPACITY AND REGULAR BOUNDARY POINTS FOR THE PARABOLIC OPERATOR OF ORDER $\alpha$
}

\author{
MASAHARU NISHIO
}

\section{§1. Introduction}

Let $R^{n+1}=R^{n} \times R$ be the $(n+1)$-dimensional Euclidean space with $n \geq 1$. We denote by $X=(x, t)$ a point in $R^{n+1}$ with $x \in R^{n}$ and $t \in R$. Consider the parabolic operator on $R^{n+1}$ :

$$
L^{(\alpha)}=\frac{\partial}{\partial t}+(-\Delta)^{\alpha}
$$

where $0<\alpha \leq 1$ and $\Delta$ denotes the Laplacian on $R^{n}$.

For a closed set $E$ in $R^{n}$, we put

$$
T^{(\alpha)}(E)=\left\{\left(s^{1 / 2 \alpha} x,-s\right) ; x \in E, s \geq 0\right\}
$$

and

$$
\Omega^{(\alpha)}(E)=R^{n+1} \backslash T^{(\alpha)}(E) .
$$

In $[\mathrm{EK}]$ and [IN], it is shown that for a non-empty open set $\omega$ in $R^{n}$, the origin $O$ is a regular boundary point of $\Omega^{(\alpha)}(\bar{\omega})$ for $L^{(\alpha)}$ (with respect to the Dirichlet problem). The purpose of this paper is to give a characterization of this type. Let $K_{2 \alpha}(x, y)$ be the kernel on $R^{n} \times R^{n}$ of the form

$$
K_{2 \alpha}(x, y)=\left\{\begin{array}{cc}
1 & \left(n=1, \alpha>\frac{1}{2}\right) \\
\max \left(0, \log \frac{1}{|x-y|}\right) & (2 \alpha=n) \\
|x-y|^{2 \alpha-n} & (1 \leq 2 \alpha<n) \\
\min \left(|x|^{2 \alpha-2},|y|^{2 \alpha-n}\right) & \left(n=1, \alpha<\frac{1}{2}\right) \\
|x-y|\left(|x-y| \theta(y, x-y)+|x-y|^{\frac{1}{2 \alpha}}\right)^{1-n-2 \alpha} & \left(n \geq 2, \alpha=\frac{1}{2}\right),
\end{array}\right.
$$

Received April 21, 1994. 
where $\theta(y, x-y)$ is the angle between $y$ and $x-y$ or $\theta(y, x-y)=0$ according as $|y||x-y| \neq 0$ or $|y||x-y|=0$. We denote by $C_{2 \alpha}(E)$ the capacity of a set $E$ associated with $K_{2 \alpha}$. Our main theorem is the following

THEOREM 1. Let $E$ be a closed set in $R^{n}$. Then the origin $O$ is a regular boundary point of $\Omega^{(\alpha)}(E)$ for $L^{(\alpha)}$ if and only if $C_{2 \alpha}(E)>0$.

From Theorem 1, it follows immediately

THEOREM 2. Let $\Omega$ be an open set in $R^{n+1}$ and $\left(x_{0}, t_{0}\right) \in R^{n+1}$ a boundary point of $\Omega$. If there exist $s_{0}>0$ and a closed set $E$ in $R^{n}$ with $C_{2 \alpha}(E)>0$ and with

$$
T_{\left(x_{0}, t_{0}\right)}^{(\alpha)}\left(E, s_{0}\right) \cap \Omega=\emptyset,
$$

where

$$
T_{\left(x_{0}, t_{0}\right)}^{(\alpha)}\left(E, s_{0}\right)=\left\{\left(x_{0}+s^{1 / 2 \alpha} x, t_{0}-s\right) ; x \in E, 0 \leq s \leq s_{0}\right\},
$$

then $\left(x_{0}, t_{0}\right)$ is a regular boundary point of $\Omega$ for $L^{(\alpha)}$.

We remark that for $1 / 2 \leq \alpha \leq 1, C_{2 \alpha}(E)$ is the $2 \alpha$-Riesz capacity of $E$. For $0<\alpha<1 / 2$, there is no relation between $C_{2 \alpha}(E)$ and the Riesz capacity of $E$ (see Section 4 for further discussions). Our typical applications of Theorem 1 is the following

Corollary 3. Let $H$ be a hyperplane in $R^{n}$ and $E$ a non-empty open subset of $H$. In the case $0 \in H$, the origin $O$ is a regular boundary point of $\Omega^{(\alpha)}(\bar{E})$ for $L^{(\alpha)}$ if and only if $1 / 2<\alpha \leq 1$. In the case $0 \notin H, O$ is a regular boundary point of $\Omega^{(\alpha)}(\bar{E})$ for $L^{(\alpha)}$ if and only if $\alpha \neq 1 / 2$.

In particular, we have

COROLlary 4. Let $n=1$ and let $E$ be a closed set in $R$. Then the origin $O$ is a regular boundary point of $\Omega^{(\alpha)}(E)$ for $L^{(\alpha)}$ if and only if

$$
\begin{array}{ll}
E \neq \emptyset & \text { for } 1 / 2<\alpha \leq 1, \\
C_{1}(E)>0 & \text { for } \alpha=1 / 2, \\
E \backslash\{0\} \neq \emptyset & \text { for } 0<\alpha<1 / 2,
\end{array}
$$

where $C_{1}(E)$ is the logarithmic capacity of $E$. 


\section{§2. Capacities and regular boundary points}

Denote by $W^{(\alpha)}$ the fundamental solution of $L^{(\alpha)}$, that is,

$$
W^{(\alpha)}(x, t)= \begin{cases}(2 \pi)^{-n} \int_{R^{n}} \exp \left(-t|\xi|^{2 \alpha}+\sqrt{-1} x \cdot \xi\right) d \xi & t>0 \\ 0 & t \leq 0\end{cases}
$$

where $x \cdot \xi$ is the inner product of $x$ and $\xi$ and $|\xi|=(\xi \cdot \xi)^{1 / 2}$. Put $\phi_{\alpha}(|x|)=$ $W^{(\alpha)}(x, 1)$. Then $\phi_{\alpha}$ is decreasing on $[0, \infty)$ and

$$
W^{(\alpha)}(x, t)=t^{-n / 2 \alpha} \phi_{\alpha}\left(t^{-1 / 2 \alpha}|x|\right)
$$

for $t>0$. Furthermore, in the case $0<\alpha<1, \phi_{\alpha}(r)$ is of order $r^{-n-2 \alpha}$ as $r \rightarrow \infty$.

Recall that for a closed set $F$ in $R^{n+1}$, the $\alpha$-parabolic capacity $\operatorname{cap}^{(\alpha)}(F)$ of $F$ is defined by

$$
\operatorname{cap}^{(\alpha)}(F)=\sup \left\{\int d \mu ; \mu \in M_{1}(F)\right\}
$$

where $M_{1}(F)$ is the set of all Radon measures $\mu \geq 0$ on $R^{n+1}$ supported by $F$ satisfying $W^{(\alpha) *} \mu \leq 1$ on $R^{n+1}$ (see [N] and [W]). If $F$ is compact, there exists a unique $\mu \in M_{1}(F)$ with $\int d \mu=\operatorname{cap}^{(\alpha)}(F)$, which is called the equilibrium measure of $F$ with respect to $W^{(\alpha)}$. For $\lambda>0$, we denote by $\tau_{\lambda}^{(\alpha)}: R^{n+1} \rightarrow R^{n+1}$ the $2 \alpha$-parabolic dilation, that is, $\tau_{\lambda}^{(\alpha)}(x, t)=\left(\lambda x, \lambda^{2 \alpha} t\right)$. By (1), we have

Proposition 5. Let $F$ be a compact set in $R^{n+1}$ and $\lambda>0$. Denote by $\mu$ and $\mu_{\lambda}$ the equilibrium measures of $F$ and $\tau_{\lambda}^{(\alpha)}(F)$ with respect to $W^{(\alpha)}$, respectively. Then we have

$$
\mu_{\lambda}=\lambda^{n} \cdot \tau_{\lambda}^{(\alpha)} \mu
$$

and

$$
\operatorname{cap}^{(\alpha)}\left(\tau_{\lambda}^{(\alpha)}(F)\right)=\lambda^{n} \operatorname{cap}^{(\alpha)}(F),
$$

where $\tau_{\lambda}^{(\alpha)} \mu$ is the image measure of $\mu$ by $\tau_{\lambda}^{(\alpha)}$.

We define the capacity associated with $K_{2 \alpha}$ in the usual manner.

Definition 1. For $E \subset R^{n}$, we put 


$$
C_{2 \alpha}(E)=\sup \left\{\int d \nu ; \nu \in \mathfrak{M}_{1}(E)\right\},
$$

where $\mathfrak{M}_{1}(E)$ is the set of all Radon measures $\nu \geq 0$ on $R^{n}$ such that $\operatorname{supp}(\nu) \subset$ $E$ and $\int K_{2 \alpha}(x, y) d \nu(y) \leq 1$ for every $x \in R^{n}$.

Definition 2 (see [IN]). Let $\Omega$ be an open set in $R^{n+1}$ and $X_{0}$ a boundary point of $\Omega$. Then $X_{0}$ is said to be regular for $L^{(\alpha)}$ (with respect to the Dirichlet problem) if

$$
\lim _{X \in \Omega, X \rightarrow X_{0}} \varepsilon_{X, \Omega \Omega}^{\prime \prime}=\varepsilon_{X_{0}} \text { (vaguely), }
$$

where $\varepsilon_{X, C \Omega}^{\prime \prime}$ is the balayaged measure of the point measure $\varepsilon_{X}$ at $X$ to $C \Omega$ (the complement of $\Omega$ ) with respect to $\tilde{W}^{(\alpha)}(x, t)=W^{(\alpha)}(x,-t)$. Denote by $\tilde{M}_{X}(C \Omega)$ the vague closure of the set of all positive Radon measures $\mu$ on $R^{n+1}$ which satisfies $\tilde{W}^{(\alpha)} * \mu \geq \tilde{W}^{(\alpha)} * \varepsilon_{X}$ on $C \Omega$. Then $\varepsilon_{X, C \Omega}^{\prime \prime}$ is the unique positive measure in $\tilde{M}_{X}(C \Omega)$ which satisfies $\tilde{W}^{(\alpha)} * \varepsilon_{X, C \Omega}^{\prime \prime} \leq \tilde{W}^{(\alpha)} * \mu$ on $R^{n+1}$ for every $\mu \in \tilde{M}_{X}(\mathrm{C} \Omega)$.

By the Wiener criterion and Proposition 5, we know the following characterization of the regular boundary points (see $[\mathrm{EG}]$ and $[\mathrm{N}]$ ).

Proposition 6. For a closed set $E$ in $R^{n}$, the following three conditions are equivalent:

(1) $O$ is a regular boundary point of $\Omega^{(\alpha)}(E)$ for $L^{(\alpha)}$.

(2) $\operatorname{cap}^{(\alpha)}\left(T^{(\alpha)}(E, s)\right)>0$ for every $s>0$.

(3) $\operatorname{cap}^{(\alpha)}\left(T^{(\alpha)}\left(E, s_{1}, s_{2}\right)\right)>0$ for some $0<s_{1}<s_{2}$,

where

$$
T^{(\alpha)}\left(E, s_{1}, s_{2}\right)=\left\{\left(s^{1 / 2 \alpha} x,-s\right) ; x \in E, s_{1} \leq s \leq s_{2}\right\}
$$

\section{§3. Proofs of Theorem 1 and Corollary 3}

In order to discuss the $\alpha$-parabolic capacity and the capacity associated with $K_{2 \alpha}$, we estimate the following integral:

$$
\int_{-2}^{-1} W^{(\alpha)}\left((x,-1)-\left((-s)^{1 / 2 \alpha} y, s\right)\right) d s
$$

Since the $2 \alpha$-Riesz kernel is the fundamental solution of $(-\Delta)^{\alpha}$, we have 
LEMma 7. For $|x| \leq 1 / 2$, there exists a constant $M>1$ such that

$$
M^{-1} R_{2 \alpha}(x) \leq \int_{-2}^{-1} W^{(\alpha)}((x,-1)-(0, s)) d s \leq M R_{2 \alpha}(x),
$$

where $R_{2 \alpha}$ is the $2 \alpha$-Riesz kernel, that is,

$$
R_{2 \alpha}(x)=\left\{\begin{array}{lll}
|x|^{2 \alpha-n} & \text { for } & 2 \alpha<n \\
\max \left(0, \log \frac{1}{|x|}\right) & \text { for } & 2 \alpha=n \\
1 & \text { for } & 2 \alpha>n
\end{array}\right.
$$

In the proof of Theorem 1, the following lemma plays an essential role.

LEMMA 8. For each $0<r_{1}<r_{2}$, there exists a constant $M>0$ such that

$$
M^{-1} K_{2 \alpha}(x, y) \leq \int_{-2}^{-1} W^{(\alpha)}\left((x,-1)-\left((-s)^{1 / 2 \alpha} y, s\right)\right) d s \leq M K_{2 \alpha}(x, y)
$$

for every $x, y \in R^{n}$ with $|x| \leq r_{2}, r_{1} \leq|y| \leq r_{2}$.

Proof. For functions $a$ and $b$ on $\left\{(x, y) ;|x| \leq r_{2}, r_{1} \leq|y| \leq r_{2}\right\}$, we write $a \approx b$ if

$$
C^{-1} b \leq a \leq C b
$$

for some constant $C \geq 1$. Assume $\alpha \geq 1 / 2$. Then we have

$$
\begin{aligned}
& \int_{-2}^{-1} W^{(\alpha)}\left((x,-1)-\left((-s)^{1 / 2 \alpha} y, s\right)\right) d s \\
= & \int_{0}^{1} s^{-n / 2 \alpha} \phi_{\alpha}\left(s^{-1 / 2 \alpha}\left|x-(s+1)^{1 / 2 \alpha} y\right|\right) d s \\
= & \int_{0}^{1} s^{-n / 2 \alpha} \phi_{\alpha}\left(\left|s^{-1 / 2 \alpha}(x-y)-s^{-1 / 2 \alpha}\left((s+1)^{1 / 2 \alpha}-1\right) y\right|\right) d s \\
\approx & \int_{0}^{1} s^{-n / 2 \alpha} \phi_{\alpha}\left(s^{-1 / 2 \alpha}|x-y|\right) d s \\
\approx & K_{2 \alpha}(x, y) .
\end{aligned}
$$

Assume $0<\alpha<1 / 2$. Then we have

$$
\int_{-2}^{-1} W^{(\alpha)}\left((x,-1)-\left((-s)^{1 / 2 \alpha} y, s\right)\right) d s
$$




$$
\begin{aligned}
& =\int_{0}^{1} s^{-n / 2 \alpha} \phi_{\alpha}\left(s^{-1 / 2 \alpha}\left|x-(s+1)^{1 / 2 \alpha} \mathrm{y}\right|\right) d s \\
& \approx \int_{0}^{1} s^{-n / 2 \alpha} \phi_{\alpha}\left(s^{-1 / 2 \alpha}\left|\left(2^{1 / 2 \alpha}-1\right) s y-(x-y)\right|\right) d s \\
& \approx \int_{0}^{1} s^{-n / 2 \alpha} \phi_{\alpha}\left(s^{-1 / 2 \alpha}|s \tilde{y}-\tilde{x}|\right) d s \quad(\text { say }=I),
\end{aligned}
$$

where $\tilde{y}=y /|y|$ and $\tilde{x}=(x-y) /\left(2^{1 / 2 \alpha}-1\right)|y|$. In the above calculation, we use the change of variables from $s$ to $s^{\prime}$ :

$$
(s+1)^{1 / 2 \alpha}=\left(2^{1 / 2 \alpha}-1\right) s^{\prime}+1 .
$$

We may assume that

$$
|\tilde{x}| \leq \min \left(\frac{1}{2},\left(\frac{\pi}{6}\right)^{\frac{2 \alpha}{1-2 \alpha}}\right)
$$

By separating into the following three cases of $\theta=\theta(\tilde{x}, \tilde{y})$, we estimate the integral $I ; \pi / 6 \leq \theta \leq \pi,|\tilde{x}|^{1 / 2 \alpha-1} \leq \theta \leq \pi / 6$ and $0 \leq \theta \leq|\tilde{x}|^{1 / 2 \alpha-1}$.

In the case $\theta \geq \pi / 6$, we have, for $0 \leq s \leq 1$,

$$
|s \tilde{y}-\tilde{x}| \approx|\tilde{x}|+s,
$$

so that

$$
\begin{aligned}
I & \approx \int_{0}^{1} s^{-n / 2 \alpha} \phi_{\alpha}\left(s^{-1 / 2 \alpha}(|\tilde{x}|+s)\right) d s \\
& \approx \int_{0}^{|\tilde{x}|} s^{-n / 2 \alpha} \phi_{\alpha}\left(s^{-1 / 2 \alpha}|\tilde{x}|\right) d s+\int_{|\tilde{x}|}^{1} s^{-n / 2 \alpha} \phi_{\alpha}\left(s^{1-1 / 2 \alpha}\right) d s \\
& \approx \int_{0}^{|\tilde{x}|} s|\tilde{x}|^{-n-2 \alpha} d s+\int_{|\tilde{x}|}^{1} s^{1-n-2 \alpha} d s \\
& \approx \begin{cases}1 & \text { for } n=1 \\
|\tilde{x}|^{2-n-2 \alpha} & \text { for } n \geq 2 .\end{cases}
\end{aligned}
$$

In the case $0 \leq \theta \leq \pi / 6$, we have

$$
|s \tilde{y}-\tilde{x}| \approx|\tilde{x}| \text { for } 0 \leq s \leq|\tilde{x}| / 3
$$

and

$$
|s \tilde{y}-\tilde{x}| \approx s \text { for } s \geq 2|\tilde{x}|
$$


so that

$$
\begin{aligned}
& \approx \int_{0}^{|\tilde{x}| / 3}+\int_{2|\tilde{x}|}^{1} s^{-n / 2 \alpha} \phi_{\alpha}\left(s^{1-1 / 2 \alpha}|s \tilde{y}-\tilde{x}|\right) d s \\
& \approx\left\{\begin{array}{cc}
1 & \text { for } n=1 \\
|\tilde{x}|^{2-n-2 \alpha} & \text { for } n \geq 2 .
\end{array}\right.
\end{aligned}
$$

In the case $|\tilde{x}|^{1 / 2 \alpha-1} \leq \theta \leq \pi / 6$, denoting by $\tilde{x} \cdot \tilde{y}$ the inner product of $\tilde{x}$ and $\tilde{y}$, we have for $|\tilde{x}| / 3 \leq s \leq 2|\tilde{x}|$,

$$
s^{-1 / 2 \alpha}|s \tilde{y}-\tilde{x}| \geq s^{-1 / 2 \alpha}|\tilde{x}| \sin \theta \approx \theta|\tilde{x}|^{1-1 / 2 \alpha} \geq 1,
$$

so that

$$
\begin{aligned}
& \int_{|\tilde{x}| / 3}^{2|\tilde{x}|} s^{-n / 2 \alpha} \phi_{\alpha}\left(s^{-1 / 2 \alpha}|s \tilde{y}-\tilde{x}|\right) d s \\
\approx & \int_{|\tilde{x}| / 3}^{2|\tilde{x}|}|\tilde{x}||s \tilde{y}-\tilde{x}|^{-n-2 \alpha} d s \\
\approx & \int_{|s-\tilde{x} \cdot \tilde{y}| \leq|\tilde{x}| \theta}|\tilde{x}|(|\tilde{x}| \theta)^{-n-2 \alpha} d s \\
& \quad+\int_{|s-\tilde{x} \cdot \tilde{y}| \leq|\tilde{x}| \theta,|\tilde{x}| / 3 \leq s \leq 2|\tilde{x}|}|\tilde{x}||s-\tilde{x} \cdot \tilde{y}|^{-n-2 \alpha} d s \\
\approx & |\tilde{x}|(|\tilde{x}| \theta)^{1-n-2 \alpha} .
\end{aligned}
$$

Therefore combining this relation with (4), in the case $|\tilde{x}|^{1 / 2 \alpha-1} \leq \theta \leq \pi / 6$, we have

$$
I \approx|\tilde{x}|(|\tilde{x}| \theta)^{1-n-2 \alpha} .
$$

In the case $\theta \leq|\tilde{x}|^{1 / 2 \alpha-1}$, we have

$$
\begin{aligned}
& \int_{|\tilde{x}| / 3}^{2|\tilde{x}|} s^{-n / 2 \alpha} \phi_{\alpha}\left(s^{-1 / 2 \alpha}|s \tilde{y}-\tilde{x}|\right) d s \\
\approx & \int_{|\tilde{x}| / 3}^{2|x|}|\tilde{x}|^{-n / 2 \alpha} \phi_{a}\left(|\tilde{x}|^{-1 / 2 \alpha}|s \tilde{y}-\tilde{x}|\right) d s \\
\approx & \int_{|s-\tilde{x} \cdot \tilde{y}| \leq|\tilde{x}|^{1 / 2 \alpha}}|\tilde{x}|^{-n / 2 \alpha} d s \\
& \quad+\int_{\left(|s-\tilde{x} \cdot \tilde{y}| \geq|\tilde{x}|^{1 / 2 \alpha}, \tilde{x}|/ 3 \leq s \leq 2| \tilde{x} \mid\right)}|\tilde{x}||s-\tilde{x} \cdot \tilde{y}|^{-n-2 \alpha} d s
\end{aligned}
$$




$$
\approx|\tilde{x}|^{(1-n) / 2 \alpha} \text {. }
$$

This and (4) imply

$$
I \approx|\tilde{x}|^{(1-n) / 2 \alpha}
$$

Thus Lemma 8 is shown by (2), (3), (5) and (6).

To prove Theorem 1, we use the following

Proposition 9. Let $\mu$ be a positive Radon measure on $R^{n} \times[0, \infty)$. For $s>0$, we put $\mu_{s}=\mu * \varepsilon_{(0, s)}$. If for any $s>0$,

$$
0 \neq\left.\mu\right|_{R^{n} \times(s, \infty)} \leq \mu_{s},
$$

then for every $0<s_{1}<s_{2}$, there exists a positive Radon measure $\nu \neq 0$ on $R^{n}$ such that

$$
\mu \geq \nu \otimes d t \quad \text { on } \quad R^{n} \times\left(s_{1}, s_{2}\right) .
$$

Here $\left.\mu\right|_{R^{n} \times(s, \infty)}$ is the restriction of $\mu$ to $R^{n} \times(s, \infty)$.

Proof. Let $\varphi \geq 0$ be a continuous function with $\operatorname{supp}(\varphi) \subset\left(s_{1}+1, s_{1}+2\right)$ and $\int \varphi(t) d t=1$. Define the positive measure $\nu$ on $R^{n}$ by

$$
\int f(x) d \nu(x)=\int f(x) \varphi(t) d \mu(x, t)
$$

for every continuous function on $R^{n}$ with compact support. Then $\nu$ is a required measure.

Applying the transformation:

$$
(\xi, \tau)=(\exp (-t) x,-a \exp (-2 \alpha t))
$$

to the measure $\mu$ in the preceding proposition, we have

Lemma 10. Let $a>0$ and $\mu \geq 0$ a measure on $R^{n} \times[-a, 0)$. If for any $0<\lambda<1$,

$$
0 \neq\left.\mu\right|_{R^{n \times\left(-a \lambda^{2 \alpha}, 0\right)}} \leq \tau_{\lambda}^{(\alpha)} \mu,
$$

then for any $-a<s_{1}<s_{2}<0$, there exists a positive Radon measure $\nu \neq 0$ on $R^{n}$ 
such that

$$
\int f d \mu \geq \iint_{s_{1}}^{s_{2}} f\left((-s)^{-1 / 2 \alpha} y, s\right) d s d \nu(y)
$$

for every continuous function $f \geq 0$ on $R^{n+1}$ with compact support.

Now we give the proofs of Theorem 1 and Corollary 3 .

Proof of Theorem 1. First suppose that $C_{2 \alpha}(E)>0$. Then there exists a positive Radon measure $\nu \neq 0$ on $R^{n}$ such that $\operatorname{supp}(\nu) \subset E$ is compact and that

$$
\int K_{2 \alpha}(x, y) d \nu(y) \leq 1
$$

for every $x \in R^{n}$. When $0<\alpha<1 / 2$, we may assume that $0 \notin \operatorname{supp}(\nu)$, because $K_{2 \alpha}(0,0)=\infty$. Define the measure $\mu$ on $R^{n+1}$ by

$$
\int f d \mu=\iint_{-2}^{-1} f\left((-s)^{-1 / 2 \alpha} y, s\right) d s d \nu(y)
$$

for every continuous function $f$ on $R^{n+1}$. By Lemma $8, W^{(\alpha)} * \mu$ is bounded on $R^{n+1}$. Hence $\operatorname{cap}^{(\alpha)}\left(T^{(\alpha)}(E, 1,2)\right)>0$. By Proposition $6, O$ is a regular boundary point of $\Omega^{(\alpha)}(E)$ for $L^{(\alpha)}$. Conversely suppose that $O$ is regular for $L^{(\alpha)}$. By Proposition 6 , we have $\operatorname{cap}^{(\alpha)}\left(T^{(\alpha)}(E, 3)\right)>0$. We may assume that $E$ is compact and that $0 \notin E$ if $0<\alpha<1 / 2$. For $s<0$, we denote by $\mu_{s}$ the equilibrium measure of $T^{(\alpha)}(E, s)$ with respect to $W^{(\alpha)}$. We remark that for any $s>0$,

$$
\left.\mu_{3}\right|_{R^{n} \times(-s, 0)} \neq 0 \text {. }
$$

For $\lambda>0$, since $\tau_{\lambda}^{(\alpha)}\left(T^{(\alpha)}(E, s)\right)=T^{(\alpha)}\left(E, \lambda^{2 \alpha} s\right)$, Proposition 5 shows $\mu_{\lambda^{2 \alpha} s}=$ $\lambda^{n} \cdot \tau_{\lambda}^{(\alpha)} \mu_{s}$. On the other hand, for $0<s_{1}<s_{2}, \mu_{s_{2}} \leq \mu_{s_{1}}$ on $T^{(\alpha)}\left(E, s_{1}\right)$ (see [N, Lemma 2.14]), so that for $0<\lambda<1$,

$$
\mu_{3} \leq \tau_{\lambda}^{(\alpha)} \mu_{3} \quad \text { on } \quad T^{(\alpha)}\left(E, 3 \lambda^{2 \alpha}\right) .
$$

By Lemma 10 , there exists a positive Radon measure $\nu \neq 0$ on $R^{n}$ such that $\operatorname{supp}(\nu) \subset E$ and that

$$
\iint_{-2}^{-1} f\left((-s)^{-1 / 2 \alpha} y, s\right) d s d \nu(y) \leq \int f d \mu_{3}
$$

for every continuous function $f \geq 0$ on $R^{n+1}$. Therefore Lemma 8 gives 


$$
\begin{aligned}
\int K_{2 \alpha}(x, y) d \nu(y) & \leq M W^{(\alpha)} * \mu_{3}(x,-1) \\
& \leq M
\end{aligned}
$$

which implies $C_{2 \alpha}(E)>0$. This completes the proof.

Proof of Corollary 3. First we remark that $\bar{E}$ has a positive $2 \alpha$-Riesz capacity if and only if $1 / 2<\alpha \leq 1$. Then in the case $O \in H$, the assertion of Corollary 3 follows from Lemma 7 . Next we assume $O \notin H$. By Theorem 1 , we have only to show $C_{2 \alpha}(\bar{E})>0$ in the case $0<\alpha<1 / 2$. We may assume that $\bar{E}$ is compact. Let $\nu$ be the restriction to $\bar{E}$ of the $(n-1)$-dimensional Lebesgue measure on $H$. Then we can show that $\int K_{2 \alpha}(x, y) d \nu(y)$ is bounded on $R^{n}$. In fact, if $x \in H$ or if $x$ and $O$ are in the same component of $R^{n} \backslash H$, then $\theta(y, x-y) \geq C_{0}$ for some constant $C_{0}>0$, so that we have

$$
\begin{aligned}
\int K_{2 \alpha}(x, y) d \nu(y) & \approx \int_{B_{H}\left(x_{0}, 1\right)}|x-y|^{2-n-2 \alpha} d \nu(y) \\
& \leq \int_{B_{H}\left(x_{0}, 1\right)}\left|x_{0}-y\right|^{2-n-2 \alpha} d \nu(y) \\
& \approx 1
\end{aligned}
$$

for $x \in R^{n}$ near $\bar{E}$, where $x_{0}$ is the nearest point in $H$ from $x$, and where $B_{H}\left(x_{0}, r\right)$ denotes the ball in $H$ with radius $r$ and center $x_{0}$. If $x$ and $O$ are not in the same component of $R^{n} \backslash H$, denote by $x_{0}$ the constant multiplication of $x$ belonging to $H$. Then

$$
\begin{aligned}
& \int_{B_{H}\left(x_{0},\left|x-x_{0}\right|, 1\right)} K_{2 \alpha}(x, y) d \nu(y) \\
\approx & \int_{B_{H}\left(x_{0},\left|x-x_{0}\right|, 1\right)}|x, y|^{2-n-2 \alpha} d \nu(y) \\
\approx & 1, \\
& \int_{B_{H},\left(x_{0},\left|x-x_{0}\right|^{1-2 \alpha},\left|x-x_{0}\right|\right)} K_{2 \alpha}(x, y) d \nu(y) \\
\approx & \int_{B_{H},\left(x_{0},\left|x-x_{0}\right|^{1-2 \alpha},\left|x-x^{0}\right|\right)}\left|x-x_{0}\right|\left|y-x_{0}\right|^{1-n-2 \alpha} d \nu(y) \\
\approx & 1,
\end{aligned}
$$

where $B_{H}\left(x_{0}, r_{1}, r_{2}\right)=B_{H}\left(x_{0}, r_{2}\right) \backslash B_{H}\left(x_{0}, r_{1}\right)$. Moreover 


$$
\begin{aligned}
& \int_{B_{H}\left(x_{0},\left|x-x_{0}\right|^{1-2 \alpha}\right)} K_{2 \alpha}(x, y) d \nu(y) \\
\approx & \int_{B_{H}\left(x_{0},\left|x-x_{0}\right|^{1-2 \alpha)}\right.}\left|x-x_{0}\right|^{(1-n) / 2 \alpha} d \nu(y) \\
\approx & 1 .
\end{aligned}
$$

Therefore we have $C_{2 \alpha}(\bar{E})>0$, which shows Corollary 3 .

\section{§4. Capacity $C_{2 \alpha}$ and the Riesz capacity}

For $0<\alpha<1 / 2$, we discuss a relation between the capacity $C_{2 \alpha}$ and the Riesz capacities. Since

$$
|x-y|^{2-2 \alpha-n} \leq K_{2 \alpha}(x, y) \leq|x-y|^{(1-n) / 2 \alpha}
$$

for $y \neq 0$ and $x \in R^{n}$ such that $|x-y|$ is sufficiently small, $C_{2 \alpha}(E)>0$ implies $C_{2-2 \alpha}^{(n)}(E)>0$ and $C_{2 \alpha}(E)=0$ implies $C_{(1-(1-2 \alpha) n) / 2 \alpha}^{(n)}(E)=0$, where $C_{\beta}^{(n)}(\cdot)$ denotes the $\beta$-Riesz capacity of $(\cdot)$ in $R^{n}$. Corollary 3 gives an example of $E$ such that $C_{2 \alpha}(E)>0$ and $C_{1}^{(n)}(E)=0$ since $C_{1}^{(n)}(H)=0$ for every hyperplane $H$ in $R^{n}$. It would be a question to be answered whether for every $\varepsilon>0$, there exists a compact set $E$ in $R^{n} \backslash\{0\}$ such that $C_{2 \alpha}(E)>0$ and $C_{2-2 \alpha-\varepsilon}^{(n)}(E)=0$. Conversely what is the infimum of $\beta$ satisfying the following condition?

There exists a compact set $E$ in $R^{n} \backslash\{0\}$ such that $C_{2 \alpha}(E)=0$ and $C_{\beta}^{(n)}(E)>0$.

Concerning the latter question, we have the following example.

Example. Let $H$ be a hyperplane in $R^{n}(n \geq 2)$ with $0 \notin H$. For every compact set $K \subset H$, we put

$$
E_{K}=\left\{s y \in R^{n} ; y \in K, 1 \leq s \leq 2\right\} .
$$

Then $C_{2 \alpha}\left(E_{K}\right)>0$ if and only if $C_{2 \alpha}^{(n-1)}(K)>0$. Choosing a generalized Cantor set $K$ whose Hausdorff dimension is equal to $n-2 \alpha-1$, we have $C_{2 \alpha}\left(E_{K}\right)=0$ and for any $\varepsilon>0, C_{2 \alpha+\varepsilon}^{(n)}\left(E_{K}\right)>0$. From this observation, the infimum of $\beta$ of the latter question is less than or equal to $2 \alpha$.

\section{REFERENCES}

[EF] E. Effros and J. Kazdan, On the Dirichlet problem for the heat equation, Indiana Univ. Math. J., 20 (1971), 683-693.

[EG] L. Evans and R. Gariepy, Wiener's criterion for the heat equation, Arch. Rational 
Mech. Anal., 78 (1982), 293-314.

[IN] M. Itô and M. Nishio, Poincaré type conditions of the regularity for the parabolic operator of order $\alpha$, Nagoya Math. J., 115 (1989), 1-22.

[N] M. Nishio, The Wiener criterion of regular points for the parabolic operator of order $\alpha$, Nagoya Math. J., 116 (1989), 163-179.

[W] N. A. Watson, Thermal capacity, Proc. London Math. Soc., 37 (1978), 342-362.

Department of Mathematics

Osaka City University

Sugimoto, Sumiyoshi

Osaka 558, Japan 